\title{
An assessment of the Microcult-GC culture test
}

\author{
P. F. UNSWORTH, H. TALSANIA, AND I. PHILliPS \\ From the Department of Microbiology, St. Thomas' Hospital, London SE1
}

\begin{abstract}
SUMMARY Microcult-GC, a miniaturised culture test, was compared with an established selective culture method for laboratory confirmation of gonorrhoea. Microcult-GC correlated $94 \%$ with the control method in identifying gonococci in male urethral specimens. Microcult-GC testing of 130 gonococci-negative vaginal swabs gave only $4.6 \%$ false-positive oxidase results, which could probably have been readily refuted by Microcult Gram stain. The test may be difficult to interpret for rectal specimens and is not appropriate for pharyngeal cultures.
\end{abstract}

\section{Introduction}

Many methods have been proposed for laboratory confirmation of the diagnosis of gonorrhoea. Microcult-GC is a miniaturised culture test for the presumptive identification of Neisseria gonorrhoeae, the performance of which requires minimal laboratory apparatus. Willcox and John (1976) made a preliminary study of the test and suggested it had potential for use in countries or areas lacking laboratory facilities and as a standard with which to compare other culture methods. We assessed the test in comparison with an established selective culture method (Phillips et al., 1972) for its ability to detect $N$. gonorrhoeae in clinical material containing the organism and for its ability to yield negative results for vaginal and faecal material which was free of $N$. gonorrhoeae.

\section{Methods}

Specimens from five groups of patients were studied. Group 1 consisted of 50 men with Gram-negative diplococci in smears of urethral pus. Group 2 consisted of 18 women attending the sexually transmitted diseases (STD) clinic, of whom eight were sexual contacts of men with gonorrhoea or had themselves been previously infected with gonorrhoea, and 10 were new patients; both cervical and urethral swabs were examined. Group 3 consisted of 131 women of childbearing age (both inpatients and outpatients) in almost all of whom gonorrhoea was not suspected but from whom high

Address for reprints: P. F. Unsworth, Cross-Infection Reference Laboratory, Central Public Health Laboratory, Colindale Avenue, London NW9 5HT

Received for publication 13 August 1978 vaginal swabs were taken and sent to the laboratory. Group 4 consisted of 12 patients in whom gonorrhoea was not suspected but from whom faecal samples were collected and sent to the laboratory. Finally, group 5 consisted of a further eight men who conformed to the criteria for group 1.

Each specimen was inoculated in the STD clinic on to a Microcult-GC culture slide and also on to another medium. The latter consisted of Oxoid blood agar base no. 2 containing $10 \%$ lysed horse blood (LBA) for vaginal swabs from patients in group 3 and LBA with colistin methosulphate $100000 \mathrm{u} / \mathrm{l}$, vancomycin $3 \mathrm{mg} / \mathrm{l}$, nystatin 12500 $\mathrm{u} / \mathrm{l}$, and trimethoprim $5 \mathrm{mg} / \mathrm{l}$ (VCNT) (Phillips et al., 1972) for samples from patients in other groups.

Microcult-GC culture slides were used according to the manufacturer's instructions. The slide and dessicant were carefully removed from each foil pouch just before use, and both culture areas of each slide were rehydrated with seven or eight drops of $4 \% \mathrm{v} / \mathrm{v}$ glycerol in water. The areas were immediately inoculated by one or other of two methods: in method $\mathrm{A}$, which was used for specimens from patients in group 2, each of the two culture areas was inoculated with a specimen from a different site; in method B, which was used for specimens from patients in other groups, the same specimen was inoculated on to both culture areas in turn. Swabs were rolled over the culture areas to give as heavy an inoculum as possible. Each Microcult slide was replaced in its foil pouch together with a $\mathrm{CO}_{2}$ generating tablet, the foil was sealed, and the slide then incubated at $37^{\circ} \mathrm{C}$.

Microcult slides inoculated by method A were incubated for 42-48 hours; both areas were then processed. Slides inoculated by method $B$ had one culture area processed after incubation for 18-24 
hours and, if the first gave negative results, the other was processed after incubation for 42-48 hours. For 29 of the 50 urethral swabs from patients in group 1, the second culture area was processed whether or not the first gave negative results in the test. Incubation of the second culture area was prolonged to four or five days in the case of urethral swabs from patients in group 5.

At the end of the period of incubation slides were processed by three different methods in quick succession: firstly, an oxidase strip was applied for five seconds to the culture area and a positive reaction was recorded if part of the area turned blue within 30 seconds; secondly, the culture area was applied aseptically to a glass slide which was later Gram-stained and examined for Gram-negative diplococci (this was carried out for only nine specimens, none of which gave positive oxidase results, from group 3, but for all specimens from the other groups); and, thirdly, the whole area was applied aseptically to LBA for groups 1, 2, 3, and 5, and to VCNT for group 4 for subculture.

Subculture plates were incubated in $10 \% \mathrm{CO}_{2}$ in humidified air at $37^{\circ} \mathrm{C}$, as were the plates on which specimens were inoculated in parallel with MicrocultGC. All plates were examined after incubation for 18-24 and 42-48 hours, and oxidase-positive colonies stained with anti-gonococcal fluorescent antibody (FA N. gonorrhoeae, Difco). Oxidasepositive organisms other than neisseriae cultured from specimens from patients in groups 3 and 4 were identified by conventional means.

Statistical comparisons were made by the $\chi^{2}$ method.

\section{Results}

All oxidase-positive diplococci cultured in the study also showed specific immunofluorescence.

Detection of $N$. gonorrhoeae by means of Microcult-GC (method B) compared with culture on VCNT are shown in Table 1 . Of 50 urethral swabs from men in group $1,48(96 \%)$ yielded positive results by culture on VCNT and a positive oxidase reaction by Microcult-GC. This was confirmed by Microcult-GC Gram stain in $45(90 \%)$ and by the isolation of $N$. gonorrhoeae on subculture from Microcult-GC in all 48. The same two swabs gave negative results by VCNT culture and Microcult-GC. The correlation between Microcult-GC (method B) and VCNT culture for patients in group 1 was $94 \%$.

The results of Microcult-GC (method A) compared with VCNT culture of urethral and cervical swabs from women in group 2 are given in Table 2. Of 18 cervical swabs six $(33 \%)$ yielded gonococci on VCNT; by Microcult-GC three of these gave positive oxidase and Gram-stain results. Of 18 urethral swabs five $(28 \%)$ yielded gonococci on VCNT; the Microcult-GC oxidase reaction was positive for four of the five and Microcult Gram-staining confirmed the presence of Gram-negative diplococci in three. Overall, subculture of Microcult-GC yielded $N$. gonorrhoeae from six out of 18 patients, from six cervical and four urethral swabs.

Table 3 refers to urethral swabs from 29 of the men in group 1 for whom a comparison was made of Microcult-GC results obtained by processing one Microcult-GC culture area after incubation for 18-24 hours or $42-48$ hours or by combining the

Table 1 Results of Microcult-GC (method B) and culture on VCNT medium for urethral swabs from 50 men with gonorrhoea

\begin{tabular}{lllll}
\hline Microcult-GC (method B) & Identification of gonococci & $\begin{array}{l}\text { Culture on VCNT medium } \\
\text { (positive result) }\end{array}$ \\
\hline Oxidase reaction & + & + & - & - \\
Gram stain & + & + & - & $2(4)$ \\
Subculture & + & $3(6)$ & $48(96)$ \\
No. of urethral swabs (\%) & $45(90)$ & & \\
\hline
\end{tabular}

+Positive - negative

Table 2 Results of Microcult-GC (method A) for specimens from 18 women attending a STD clinic*

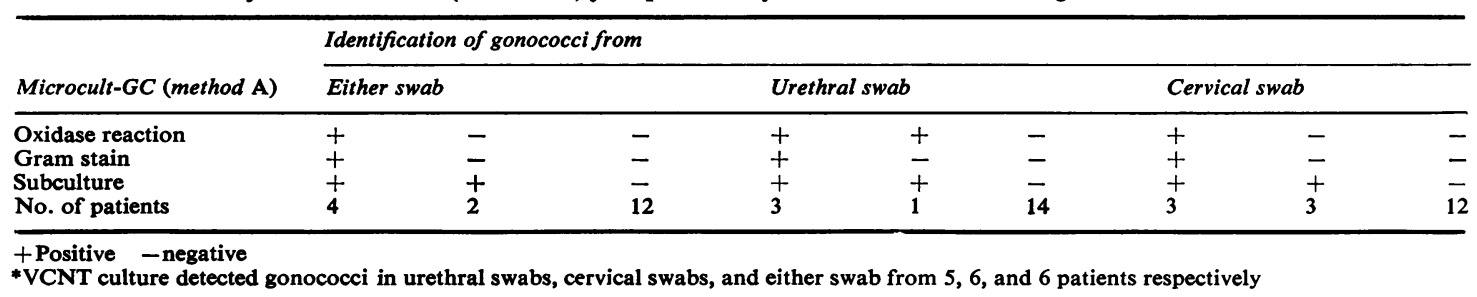


Table 3 Effect of duration of incubation on Microcult-GC in 29 men with gonorrhoea*

\begin{tabular}{|c|c|c|c|c|c|c|c|c|c|c|c|c|c|}
\hline \multirow{4}{*}{$\frac{\text { Microcult-GC }}{\text { Oxidase reaction }}$} & \multicolumn{13}{|c|}{ Results after incubation of } \\
\hline & \multicolumn{11}{|c|}{ One culture area for (hours) } & \multirow{2}{*}{\multicolumn{2}{|c|}{$\begin{array}{l}\text { One culture area for } 18-24 h \\
\text { and one area for } 42-48 h \\
(\text { method } B)\end{array}$}} \\
\hline & \multicolumn{5}{|c|}{$18-24$} & \multicolumn{6}{|c|}{$42-48$} & & \\
\hline & + & + & - & - & - & + & + & + & + & - & - & $+\quad+$ & - \\
\hline Gram stain & + & - & + & - & - & + & + & - & - & + & - & $+\quad-$ & - \\
\hline Subculture & + & + & + & + & - & + & - & + & - & - & - & $+\quad+$ & - \\
\hline No. of urethral swabs & 23 & 1 & 1 & 1 & 3 & 16 & 4 & 4 & 2 & 1 & 2 & 252 & 2 \\
\hline
\end{tabular}

+Positive - negative

*Urethral swabs yielded gonococci on VCNT medium after incubation for 18-24 h, $42-48$ h, and for either period in 25,2 , and 27 patients respectively

two (method B). Method B yielded more positive results by oxidase reaction, Gram stain, and subculture than did processing one area after either 18-24 hours or 42-48 hours; similarly oxidase and Gram-stain results were more often both positive for the same specimen; differences were not significant ( $P>0.05$ in all comparisons). The MicrocultGC Gram stain showed red amorphous material in the absence of Gram-negative diplococci, which counted as a positive result, from five of 29 areas incubated for $42-48$ hours, but from only one of 29 areas incubated for 18-24 hours.

All eight urethral swabs from patients in group 5 gave positive Microcult-GC oxidase, Gram-stain, and subculture results when processed after incubation for 18-24 hours. For three swabs the second Microcult culture area was processed after three days' incubation; the Gram stain of all three showed red amorphous material. In one case there were also occasional pairs of Gram-negative diplococci, and subculture of this specimen yielded gonococci. Subculture of the second specimen, and incubation for 48 hours, yielded two colonies of $N$. gonorrhoeae; and the third gave negative results on subculture. For five swabs the second Microcult culture area was processed after five days' incubation, at which time the Gram-stain results were: one positive, one negative, and three showing only red amorphous material. Subculture gave positive results only from the specimen with Gram-negative cocci in the Gram stain.

Of the 131 high vaginal swabs from patients in group 3 cultured directly on to lysed blood agar, nine yielded oxidase-positive organisms; six were pseudomonads and three were yeasts. By MicrocultGC (method B) seven swabs gave a positive oxidase reaction; Microcult-GC Gram staining was not performed on these, but subculture of one swab yielded $N$. gonorrhoeae and of the other six Pseudomonas aeruginosa or Pseudomonas species. The single case of gonorrhoea detected by Microcult-GC was not detected by culture on LBA, but gonococci had been previously isolated from the patient by culture on VCNT.

Culture of 12 faecal specimens on VCNT yielded non-gonococcal oxidase-positive colonies in five cases. Microcult-GC (method B) yielded a positive oxidase reaction for two of them; Pseudomonas aeruginosa grew on subculture from one oxidasepositive specimen and a Bacillus species from the second.

\section{Discussion}

The principle of Microcult-GC is presumptive identification of $N$. gonorrhoeae by means of growth on selective medium, oxidase reaction, and Gram stain. The test would not distinguish either meningococci-though these occur only rarely in genital specimens (Faur et al., 1975) - or other neisseriae, so that it could not be reliably used for pharyngeal specimens. Microcult-GC requires only sterile swabs, an incubator, Gram-staining equipment, and a microscope. Culture slides can be stored at room temperature $\left(<30^{\circ} \mathrm{C}\right)$ for months.

A very faint blue tinge sometimes appeared within 30 seconds of applying the oxidase strip on culture areas not containing organisms, but with a little practice this was easily recognised as a negative reaction. Gram-staining of Microcult culture areas occasionally showed red amorphous material, apparently due to degenerate gonococci; its colour was identical to that of typically-stained gonococci and it was never seen in specimens with completely negative results. Prolonged incubation was associated with an increased frequency of red amorphous material and with decreased gonococcal viability on subculture. It is necessary to confirm positive oxidase results by Gram staining as various bacteria may produce these. Gram stains usually showed clear-cut Gram-positive and Gram-negative cocci and bacilli or the red amorphous material. 
Unlike Williams et al. (1978) we found that subculture from Microcult-GC was easy and the results correlated completely with those of VCNT culture. Our method of subculture delivered a heavier inoculum and we minimised delay after oxidase-testing. Subculture could be used for biochemical or immunofluorescent tests and antimicrobial sensitivity-testing.

The rate of detection of gonococci from male urethral swabs by Microcult-GC was high; paired oxidase-positive and Gram-stain results were falsely negative in only $6 \%$ of cases. This could be remedied by subculturing all areas immediately after Gram staining, but this defeats the object of simplicity. Microcult-GC was less effective in detecting gonococci in female specimens but in these instances the inocula on Microcult may have been smaller than those on VCNT and the groups were too small for a complete assessment.

Microcult-GC false-positive oxidase reactions occurred with only $4.6 \%$ of high vaginal swabs. Microcult Gram staining would probably have given negative results for these specimens, which yielded pseudomonads on subculture. False-positive oxidase reactions were found for two out of 12 faecal specimens tested; one of these gave a negative result in the Microcult Gram stain, but the other result was difficult to interpret. Difficulties might be expected in using the Microcult test for rectal specimens, especially if there was heavy faecal contamination.
We agree with Willcox and John (1976) and Williams et al. (1978) that Microcult-GC may be useful in areas or countries which lack laboratory facilities. In addition, the test allows subculture of gonocococci without the need to incorporate antibiotics into the medium used, which might be an advantage in some laboratories. The detection rate of gonococci in specimens with positive results from men is high and the false-positive rate for vaginal specimens is low. Our limited experience suggests that the method may be less sensitive for specimens from women with gonorrhoea and may yield results from faecally contaminated samples that are difficult to interpret.

We thank Dr C. Nicol, from whose patients swabs were obtained, and Mr I. Williams and Sister I. Rikof for help in obtaining these specimens. We thank Mr R. Newall of the Ames Company for kindly supplying Microcult-GC tests.

\section{References}

Faur, Y. C., Weisburd, M. H., and Wilson, M. E. (1975). Isolation of Neisseria meningitidis from the genito-urinary tract and anal canal. Journal of Clinical Microbiology, 2, 178-182.

Phillips, I., Humphrey, D., Middleton, A., and Nicol, C. (1972). Diagnosis of gonorrhoea by culture on a selective medium containing vancomycin, colistin, nystatin, and trimethoprim (VCNT). British Journal of Venereal Diseases, 48, 287-292.

Willcox, R. R., and John, J. (1976). Simplified method for the cultural diagnosis of gonorrhoea. British Journal of Venereal Diseases, 52, 256.

Williams, R. J., Ratnatunga, C. S., Hamilton-Miller, J. M. T., and Brumfitt, W. (1978). Evaluation of the Microcult system for isolating and identifying Neisseria gonorrhoeae. Journal of Clinical Pathology, 31, 209-212. 